\title{
Ruptured abdominal aortic aneurysm treated with early REBOA and open abdominal management with applied shoelace technic
}

\author{
Ginga Suzuki $^{1}$, Ryo Ichibayashi ${ }^{1}$, Saki Yamamoto ${ }^{1}$, Hibiki Serizawa ${ }^{1}$, Takahide Yao ${ }^{2}$, Toru \\ Kameda $^{2}$, Yoshimi Nakamichi ${ }^{1}$, Masayuki Watanabe ${ }^{1}$, Hiroshi Masuhara ${ }^{2}$, and Mitsuru \\ Honda $^{1}$ \\ ${ }^{1}$ Toho University Omori Medical Center \\ ${ }^{2}$ Toho University
}

November 6, 2020

\begin{abstract}
We report a successful case of ruptured abdominal aortic aneurysm (rAAA) treated with endovascular aortic repair (EVAR) following resuscitative endovascular balloon occlusion of the aorta (REBOA) and treated with devised open abdominal management (OAM) for abdominal compartment syndrome (ACS). We used an applied shoelace technique with vacuum pack closure.
\end{abstract}

Article type: Case report

Title: Ruptured abdominal aortic aneurysm treated with early REBOA and open abdominal management with applied shoelace technic

Running title: RAAA treated with REBOA and devised OAM

Authors: Ginga Suzuki ${ }^{1}$, Ryo Ichibayashi ${ }^{1}$, Saki Yamamoto ${ }^{1}$, Hibiki Serizawa ${ }^{1}$, Takahide Yao ${ }^{2}$, Toru Kameda $^{2}$, Yoshimi Nakamichi ${ }^{1}$, Masayuki Watanabe ${ }^{1}$, Hiroshi Masuhara ${ }^{2}$, Mitsuru Honda ${ }^{1}$

Institution: 1. Critical Care Center, Toho University Omori Medical Center, 6-11-1 Omori-nishi, Ota-ku, Tokyo 143-8541, Japan

2. Division of Cardiovascular Surgery, Department of Surgery, School of Medicine, Faculty of Medicine, Toho University, 6-11-1 Omori-nishi, Ota-ku, Tokyo 143-8541, Japan

Corresponding author: Ginga Suzuki (ginga.suzuki@med.toho-u.ac.jp)

Critical Care Center, Toho University Omori Medical Center

6-11-1 Omori-nishi, Ota-ku, Tokyo 143-8541, Japan

Tel 03-3762-4151, FAX 03-3762-4129

Key words: ruptured abdominal aortic aneurysm, open abdominal management, REBOA, abdominal compartment syndrome

Key Clinical Message

The resuscitative endovascular balloon occlusion of the aorta and and devised open abdominal management may effective for treatment of abdominal compartment syndrome due to ruptured abdominal aortic aneurysm. 
This study is reported with the consent of the patient.

Abstract

We report a successful case of ruptured abdominal aortic aneurysm (rAAA) treated with endovascular aortic repair (EVAR) following resuscitative endovascular balloon occlusion of the aorta (REBOA) and treated with devised open abdominal management (OAM) for abdominal compartment syndrome (ACS). We used an applied shoelace technique with vacuum pack closure.

Introduction

Ruptured abdominal aortic aneurysm (rAAA) is a serious condition with a high fatality rate $^{1,2}$, and urgent attention is needed. It is desirable to stop the bleeding as soon as possible, but it takes a certain time to transfer to the operating room, and shock and cardiac arrest may occur during that time. Therefore, insertion of resuscitative endovascular balloon occlusion of the aorta (REBOA) may be attempted at the initial treatment ${ }^{1,2}$. It is less invasive than surgical aortic occlusion, and previous researches report its effectiveness ${ }^{1,2}$.

In addition, endovascular aortic repair (EVAR) following REBOA may be effective and less invasive than artificial vessel replacement. However, inability to remove the hematoma can lead to abdominal compartment syndrome..$^{2,3}$

In the present case, we diagnosed ruptured abdominal aortic aneurysm and performed EVAR with REBOA support. And we treated postoperative abdominal compartment syndrome with applied shoelace technic as open abdominal management (OAM).

This study has been approved by Toho University Omori Medical Center ethical committee.

Case report

The patient was 76-year-old man. No significant medical history or family history. His family found him lying down in the bathroom at home and called an ambulance.

His vital signs on arrival were as follows: Glasgow Coma Scale was E3V4M6, body temperature was 35.9, blood pressure was unmeasurable, heart rate was 93 , respiratory rate was 22 , in addition, $\mathrm{SpO}_{2}$ was unmeasurable. An abdominal echo was performed and we recognized an abdominal aortic aneurysm and a relatively high-intensity soft tissue shadow around it. On arrival, he complained of abdominal pain, and was diagnosed as ruptured abdominal aortic aneurysm. Rapid infusion was performed from the peripheral routes, and a vascular sheath was quickly inserted into the right femoral artery and the left brachial artery and transformed to the fluoroscopy room. REBOA (Rescue Ballon?, Tokai Medical Products, Aichi, Japan) was inserted through the sheath of the right femoral artery. While seeing through the fluoroscopy, the guide wire was carefully inserted and inflated at the level of Th12. An increase in blood pressure in the upper limbs was confirmed (93/66 $\mathrm{mmHg}$ ). After that, enhanced CT was performed (Figure 1) and he was transformed to the operating room. A $55 \times 68 \mathrm{~mm}$ aortic aneurysm was found from just below the bifurcation of the renal artery to just above the bifurcation of the common iliac artery, and retroperitoneal blood and leakage of contrast medium were observed. The blood test findings on arrival are shown in Table 1.

After the introduction of anesthesia, the REBOA was replaced with Reliant? balloon catheter (Medtronic Japan Co., Ltd., Tokyo, Japan) to occlude the aorta at the level just above the celiac artery. Next to the occlusion, an aortography was performed by inserting a pig tail catheter from the left femoral artery. The rupture area was unknown, but it was confirmed that EVAR was possible. GORE?EXCLUDER?C3? (W.L. Gore \& Associates, inc. , Delaware, United States) was selected as the device. After the placement of the Trunk-Ipsilateral Leg Endoprosthesis? (W.L. Gore \& Associates, inc. , Delaware, United States), it was confirmed that there was no endoleak after adjusting the position. Subsequently, the intravesical pressure was found to be $25 \mathrm{mmHg}$, and an increase in intraabdominal pressure was suspected. A midline abdominal incision from just below the xiphoid process to just above the pubic symphysis was performed to 
prevent abdominal compartment syndrome (ACS). After washing the abdominal cavity, temporary closure was performed with a vacuum pack. ${ }^{4}$

After returning to the ICU, blood products were transfused to stabilize breathing and circulation. Water removal by hemodialysis was started on the third day of hospitalization. On the 3rd day of hospitalization, a temporary closure was opened to confirm hemostasis, and the abdominal cavity was observed. Although hemostasis was confirmed, it was judged that intestinal edema was so strong that he could not close the abdomen. Subsequent closure of the abdomen was expected to be difficult, so another vacuum pack closure was performed to prevent shortening of the peritoneum, subcutaneous tissue, and skin. By applying the shoelace technique, ${ }^{5}$ the peritoneum, subcutaneous tissue, and skin were lumped together through a vessel cotton tape and pulled toward the opposite side. A vacuum pack closure was added above the shoelace and temporarily closure was performed again (Figure 2). After that, the water was removed, and a laparotomy closure was performed on the 6th day of hospitalization, although some intestinal edema remained. ACS did not come after the operation.

Postoperatively, septic shock was accompanied by ventilator-related pneumonia, but he was successfully recovered. A tracheotomy was performed on the 17th day of hospitalization, but rehabilitation was started and he was transferred to the general ward when he was able to sitting at the bed edge with assistance.

\section{Discussion}

It is considered that there were two important points that saved the present case. First, rapid diagnosis, rapid infusion and balloon occlusion were performed. Second, it was possible to close the abdomen relatively early by performing OAM applying the shoelace technique ${ }^{5}$ when the hemostasis was confirmed.

Regarding the first point, we diagnosed by abdominal echo, performed rapid fluid infusion from 3 peripheral routes of $18 \mathrm{G}$, and placed REBOA prior to CT. As a result, the occlusion was achieved in 35 minutes from arrival. Lifesaving is difficult if cardiac arrest occurs immediately after arrival, and left thoracotomy and direct aortic occlusion may be selected. In the present case, although the shock persisted, rapid infusion primarily prevented cardiac arrest, and the shock could be removed with a minimally invasive method with REBOA. This treatment was a good bridge for EVAR performed later, and it is considered that the treatment was less invasive than the performed by thoracotomy and laparotomy with artificial vessel. Regarding the REBOA access, it has been reported that it is able to be inserted from the left brachial artery, ${ }^{6,7}$ but we use the left or right femoral artery as the first choice and the left upper brachial artery as the second choice. This is because access from the left brachial artery, the risk of peripheral emboli from the aortic arch or the descending aorta is higher than with the femoral approach. ${ }^{7}$ It may be difficult to insert the REBOA from the femoral artery depending on the meandering of the artery or aorta. In that case, the access is switched to the left brachial artery. Also in the present case, the REBOA procedure was performed after simultaneously inserted sheaths in both the femoral and the brachial artery.

Regarding the second point, in the present case we carried out a devised vacuum pack closure in consideration that the peritoneum, subcutaneous tissue, and skin may contract and become unable to close. Although the usual vacuum pack closure was performed in the first surgery, it was expected that the intestinal edema would be strong and the laparotomy closure would be difficult in the second observation. In order to prevent from shortening the peritoneum, subcutaneous tissue, and skin as much as possible, vacuum pack closure was performed by passing a bunch of skin from the peritoneum through a vessel tape and pulling the abdominal wall in a manner similar to the shoelace technique. on the 6th day of hospitalization, intestinal edema was still present, but we were able to close the abdomen. The abdomen was closed relatively early considering the infection of the stent graft. We think that the laparotomy closure was performed at an appropriate time because he passed without ACS. This is partly because the applied shoelace technique prevented the shortening of the peritoneum and skin. We considered that this method may be useful in OAM where it is expected to be difficult to close.

Conclusion 
Abdominal echo is useful for rapid diagnosis of rAAA. If shock persists even after rapid infusion, rapid REBOA before CT may prevent cardiac arrest. In OAM for ACS, an applied shoelace technique may be advantageous for the secondary abdominal closure.

Disclosure Statement

Conflict of Interest: None declared

Author Contributions

Study conception: GS

Data collection: GS, RI

Writing: GS, RI, TY, HM

Critical review and revision: all authors

Final approval of the article: all authors

Accountability for all aspects of the work: all authors

Ethics approval

We got the patient's family consent for this report.

Funding information

Not applicable.

Acknowledgments

The authors are greatly indebted to all the personnel at the Critical Care Center, Toho University Omori Medical Center.

References

1. Borger van der Burg BLS, van Dongen TTCF, Morrison JJ, et al. A systematic review and metaanalysis of the use of resuscitative endovascular balloon occlusion of the aorta in the management of major exsanguination. Eur J Trauma Emerg Surg. 2018; 44: 535-50.

2. Aoki C, Kondo N, Saito Y, et al. Improved Outcomes for Ruptured Abdominal Aortic Aneurysms Using Integrated Management Involving Endovascular Clamping, Endovascular Replacement, and Open Abdominal Decompression. Ann Vasc Dis. 2017; 10: 22-8.

3. Ersryd S, Gidlund KD, Wanhainen A, et al. Editor's Choice - Abdominal Compartment Syndrome after Surgery for Abdominal Aortic Aneurysm: Subgroups, Risk Factors, and Outcome. Eur J Vasc Endovasc Surg. 2019; 58: 671-9.

4. Barker DE, Kaufman HJ, Smith LA, et al. Vacuum pack technique of temporary abdominal closure: a 7-year experience with 112 patients. J Trauma. 2000; 48: 201-6.

5. Harris I. Gradual closure of fasciotomy wounds using a vessel loop shoelace. Injury. 1993; 24: 565-6.

6. Raux M, Marzelle J, Kobeiter H, et al. Endovascular balloon occlusion is associated with reduced intraoperative mortality of unstable patients with ruptured abdominal aortic aneurysm but fails to improve other outcomes. J Vasc Surg. 2015; 61: 304-8.

7. Matsuda H, Tanaka Y, Hino Y, et al. Transbrachial arterial insertion of aortic occlusion balloon catheter in patients with shock from ruptured abdominal aortic aneurysm. J Vasc Surg. 2003; 38: $1293-6$.

Figure Legends

Figure 1. The enhanced CT scan on admission. 
The white arrow and arrowhead represent the celiac artery and resuscitative endovascular balloon, respectively. The balloon is deflated in half. A large amount of blood is found in the retroperitoneal cavity.

Figure 2. The vacuum pack closure whit an applied shoelace technique.

The peritoneum, subcutaneous tissue, and skin were lumped together through a cotton tape and pulled toward the opposite side

\section{Hosted file}

Table 1.pdf available at https://authorea.com/users/373224/articles/491201-rupturedabdominal-aortic-aneurysm-treated-with-early-reboa-and-open-abdominal-management-withapplied-shoelace-technic

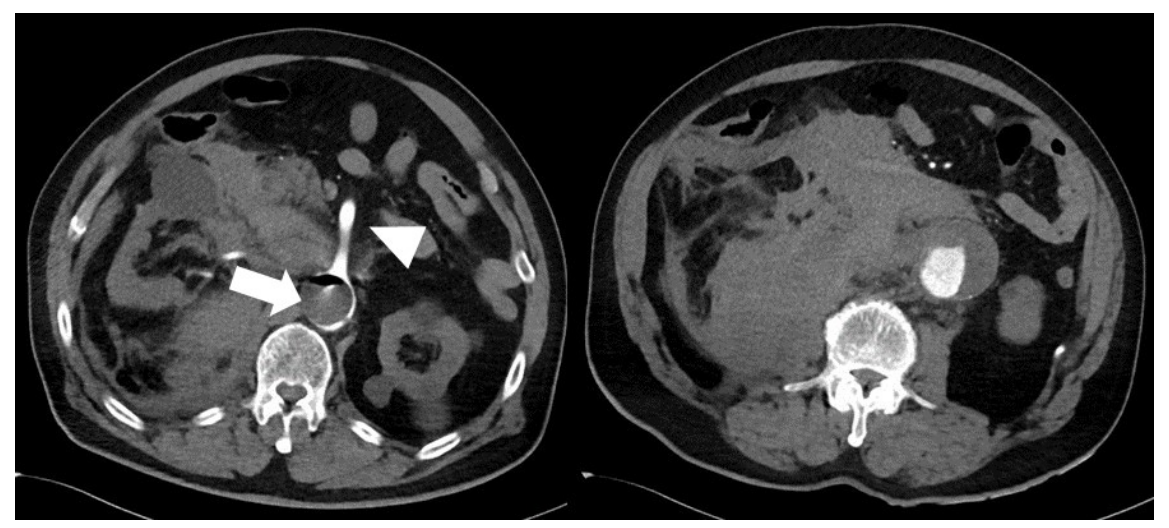




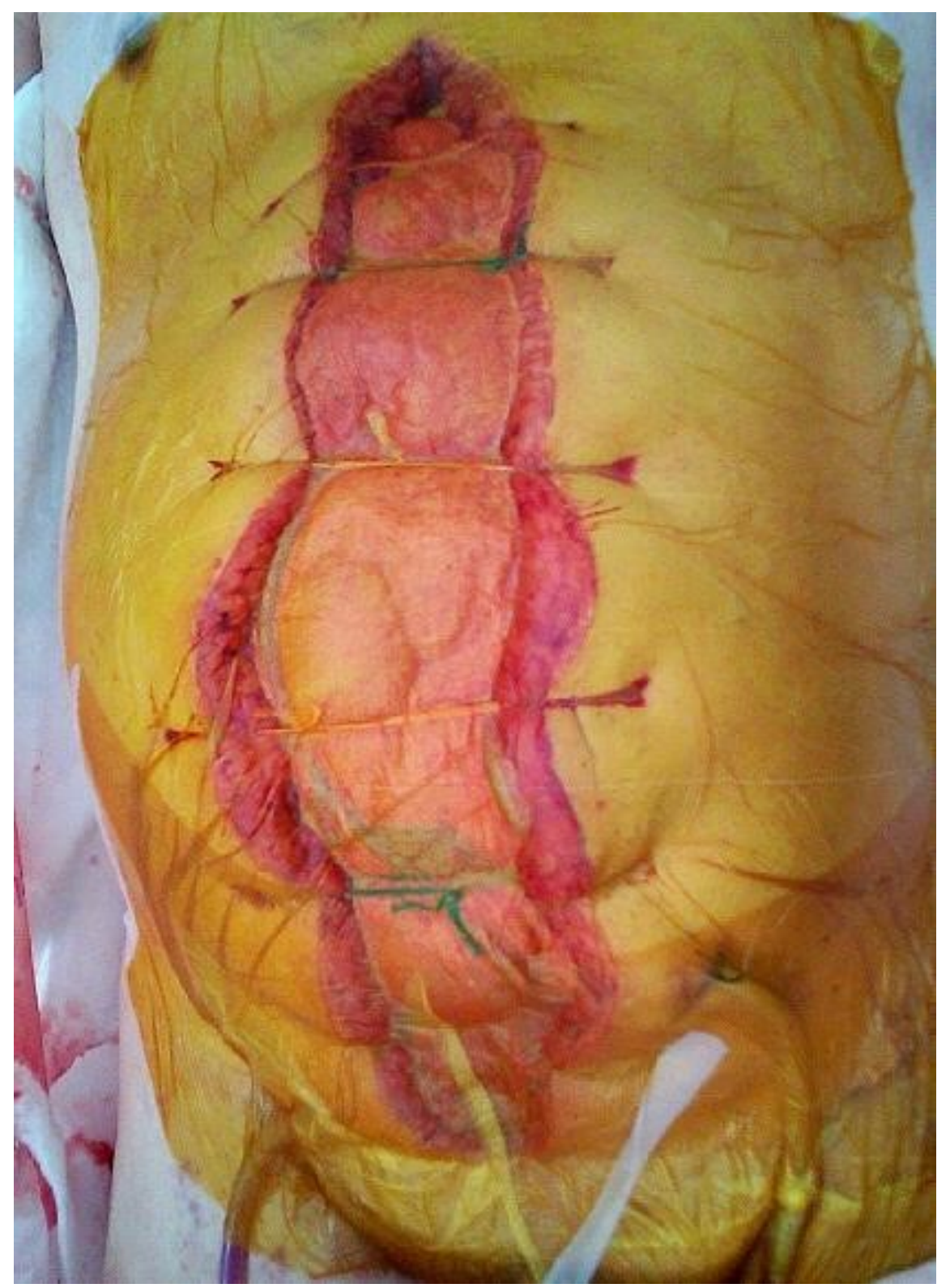

